\title{
Evaluación y manejo de la refractariedad plaquetaria: una propuesta
}

\section{Evaluation and management of platelet refractoriness: a proposal}

\author{
Dra. Alejandra Calderón Gasca,* Dr. Noiver Graciano Vera*
}

\begin{abstract}
Resumen
Introducción: La refractariedad plaquetaria se define como un incremento plaquetario postransfusión menor al esperado, se presenta en $27 \%$ de los pacientes a los que se les realiza transfusión plaquetaria, lo que constituye una limitación a la hora de transfundir un paciente con esta condición. Objetivo: Proponer un enfoque práctico para la evaluación y el manejo de los pacientes con refractariedad plaquetaria. Se sugiere un algoritmo para su tratamiento. Material y métodos: Se realizó una búsqueda de referencias bibliográficas actualizadas sobre el tema de interés en la base de datos MEDLINE, a través del motor de búsqueda PubMed. Conclusiones: El manejo de la refractariedad plaquetaria inicia con la prevención de la sensibilización. A la fecha no existe un método para diagnosticar de forma precisa la refractariedad de origen no inmune, y en muchas ocasiones puede coexistir con algún grado de refractariedad inmune.
\end{abstract}

Palabras clave: Refractariedad plaquetaria, recuento plaquetario, transfusión plaquetaria, aloinmunización.

\section{Abstract}

Introduction: Refractoriness to platelet transfusion therapy can be defined as post-transfusion platelet increment that is less than expected, platelet refractoriness occurs in $27 \%$ of patients who undergo a platelet transfusion, constituting a limitation when transfusing a patient with this condition. Objective: Design a pragmatic approach to the evaluation and management of patients with platelet refractoriness. We present an algorithm for their management. Material and methods: The authors conducted a search of references of recent articles addressing the subject of interest in the database MEDLINE, through PubMed. Conclusions: The management of platelet refractoriness begins with the prevention of sensitization. To date there is no method to diagnose refractoriness of non-immune origin, in many cases it can coexist with some degree of immune refractoriness.

Keywords: Platelet refractoriness, platelet count, transfusion, alloimmunization.

\section{Introducción}

* Servicio de Hematología y Cirugía Pediátrica. Instituto Nacional de Cancerología. Bogotá, Colombia.

Citar como: Calderón GA, Graciano VN. Evaluación y manejo de la refractariedad plaquetaria: una propuesta. Rev Mex Med Transfus. 2020; 13 (1): 7-14. doi: 10.35366/95494

Rev Mex Med Transfus 2020; 13 (1): 7-14

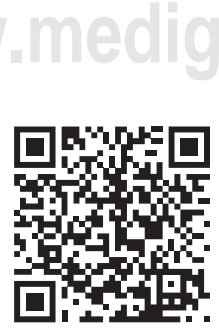

Desde la primera demostración en 1910 por W. W. Duke de la mejoría de una enfermedad hemorrágica al realizar una transfusión de plaquetas en tres individuos con trombocitopenia, la transfusión de plaquetas se ha convertido en el estándar del tratamiento en los pacientes trombocitopéni- 
cos. En la actualidad, este es el hemocomponente que se transfunde con mayor frecuencia.,2

La refractariedad plaquetaria se define como un incremento plaquetario postransfusión menor al esperado. ${ }^{3,4}$ La refractariedad plaquetaria se presenta en $27 \%$ de los pacientes a los que se les realiza transfusión plaquetaria, lo que constituye un reto y una limitación a la hora de transfundir a un paciente con esta condición.

El objetivo de este artículo fue diseñar y proponer un enfoque práctico para la evaluación y el manejo de los pacientes con refractariedad plaquetaria. Se sugiere un algoritmo para su tratamiento.

\section{Material y métodos}

Se realizó una búsqueda durante seis meses de referencias bibliográficas actualizadas sobre el tema de interés en la base de datos MEDLINE, a través de la base de datos PubMed, usando palabras claves y combinando diferentes filtros de búsqueda. Luego, se realizó la selección de los artículos y se descargaron usando las herramientas de la biblioteca del Instituto Nacional de Cancerología.

\section{Desarrollo}

El estudio para reducir la aloinmunización plaquetaria (Trial to Reduce Alloimmunization to Platelets [TRAP]), terminado en 1997, ${ }^{5}$ define la refractariedad plaquetaria como un incremento corregido de plaquetas $(\mathrm{CCl}$, por sus siglas en inglés) a la hora de postransfusión menor a $5 \times$ 10\%/L en dos episodios de transfusión consecutivos, usando plaquetas ABO compatibles y frescas, con un tiempo de almacenamiento menor a 48 horas, siendo ésta la definición aceptada de forma generalizada, incluyendo a la American Society of Clinical Oncology. ${ }^{6-8}$

En las condiciones actuales la viabilidad de la mayoría de las unidades plaquetarias a trans- fundir oscila en cinco días. Existen parámetros para evaluar la viabilidad de las plaquetas, entre éstos los más usados son el pH que se espera que esté entre 6.2 y 7.4 , la temperatura alrededor de $22{ }^{\circ} \mathrm{C}$ y la presencia de remolinos al agitar los concentrados plaquetarios (swirling).5,9,10 Sin embargo, no es una definición práctica en algunas ocasiones porque requiere el conteo plaquetario del producto infundido, que de forma rutinaria no está disponible." Para el propósito de realizar este cálculo, se asume que cada unidad de plaquetas por aféresis contiene $3 \times 10^{11}$ plaquetas y en cada unidad derivada de sangre total el concentrado contiene $5.5 \times 10^{10}$ plaquetas. $^{\text {? }}$

La refractariedad plaquetaria se clasifica según la causa: inmunes y no inmunes, que corresponden al 20 y $80 \%$ de los casos, respectivamente. Dentro de las causas inmunes se considera solamente la presencia de anticuerpos antiHLA-1 o antiplaquetarios, excepto los pacientes con diagnóstico de púrpura trombocitopénica idiopática (PTI) o de enfermedad autoinmune o asociada a medicamentos (Tabla 7).12

Dada la mayor frecuencia de causas no inmunes es necesario descartar que se trate de éstas en primera instancia, para lo que se recomienda un estudio inicial con hemograma, PT, dímero $D$, ecografía abdominal con el fin de descartar causas no inmunes como hipertensión portal y esplenomegalia, productos de la degradación de la fibrina y extendido de sangre periférica y función renal. ${ }^{3}$

Existen factores no inmunes que afectan el incremento plaquetario postransfusional que deben ser considerados en todo paciente a transfundir, como una mayor área de superficie corporal, sexo masculino, esplenomegalia y la presencia de hipertensión portal. ${ }^{14-16}$

A la fecha no existe un método para diagnosticar de forma precisa la refractariedad de origen no inmune, y en muchas ocasiones puede coexistir con algún grado de refractariedad inmune. Es más característico que se presente 
un descenso en el conteo plaquetario de manera tardía alrededor de la hora 18 a 24 postransfusión. ${ }^{16}$

En cuanto al manejo de la refractariedad no inmune, debe establecerse la causa de base y tratarla. Y en el caso de presencia de autoanticuerpos, el uso de terapia inmunosupresora es importante, en contraste con la refractariedad inmune, en la cual el uso de terapia inmunosupresora no ha demostrado un rol relevante. ${ }^{14,17}$

\section{Refractariedad plaquetaria inmune}

La refractariedad inmune se desarrolla por aloanticuerpos dirigidos contra antígenos del sistema HLA-1 y antígenos plaquetarios. Los antígenos HLA A y B explican $80 \%$ de los casos, los antígenos plaquetarios de 5 a 10\% y las causas mixtas explican el resto. La presencia de estos anticuerpos es una causa necesaria, pero no suficiente, por lo que siempre es necesario ante su evidencia, confirmar el descenso plaquetario. $15,18,19$

El antecedente de embarazo constituye un factor de riesgo para el desarrollo de aloanticuerpos, se han encontrado hasta en $4 \%$ de las mujeres que han tenido una gestación y en $26 \%$ de las mujeres que han tenido cuatro gestaciones. Otro grupo de riesgo es el de los pacientes politransfundidos y con enfermedades hematooncológicas, la presencia de anticuerpos y refractariedad inmune se puede ver desde 40 a $100 \%$ de los casos. Por lo que este grupo de pacientes, ante la posibilidad de necesitar soporte transfusional de forma crónica, siempre deben tener un abordaje particular., $5,20-22$

El manejo de la refractariedad plaquetaria inicia con la prevención de la sensibilización. Es fundamental considerar la compatibilidad ABO, mas no la RH. Cuando esto no se lleva a cabo existe el riesgo de desarrollar refractariedad plaquetaria hasta en $20 \%$ de los casos, el inicio de esto es el desarrollo de anticuerpos a las ocho semanas después de la transfusión de plaquetas no compatibles, sin relación dosis-efecto, es decir, con sólo una transfusión puede iniciarse la producción de anticuerpos. ${ }^{23}$

Otra medida que ha mostrado disminuir la posibilidad de sensibilización plaquetaria de $45 \%$ a $17-21 \%$ es la transfusión de unidades leucorreducidas (< 0.01\%), lo que sólo puede ser posible al filtrar las unidades o al exponerlas a una irradiación de 20 Gy. ${ }^{5}$

En Canadá, en los años 90, un estudio realizado posterior a la introducción de la leucorreducción universal mostró una reducción en la incidencia

Tabla 1: Causas de refractariedad plaquetaria.

Inmunes

Aloanticuerpos plaquetarios

- Antígenos leucocitarios humanos (HLA)

- Antígenos plaquetarios humanos (HPA)

- $\mathrm{ABO}$

Otros anticuerpos

- Autoanticuerpos plaquetarios

- Anticuerpos plaquetarios dependientes de medicamentos Complejos inmunes
No inmunes

Infección

Fiebre

Antibióticos, antifúngicos

Heparina

Sangrado

Enfermedad injerto contra huésped

Coagulación intravascular diseminada (CID)

Enfermedad veno-oclusiva

Esplenomegalia, hiperesplenismo

Aumento de peso

Gestaciones múltiples 
de aloinmunización (19 vs 7\%) y refractariedad plaquetaria (14 vs 4\%) en pacientes transfundidos crónicamente. Es de resaltar que la leucorreducción debe ser realizada una vez se extraiga la unidad de plaquetas del donante, en la etapa prealmacenamiento, ya que es posible que al hacerla después del almacenamiento se presenten inconvenientes con la vida útil del filtro y que éste no funcione apropiadamente. ${ }^{24}$

Hasta el momento no se ha demostrado que haya un impacto en transfundir unidades por aféresis versus usar pool de plaquetas en cuanto a la sensibilización. ${ }^{25}$ Sin embargo, en muchos centros las aféresis plaquetarias sólo se realizan a donantes masculinos o a mujeres que no hayan tenido gestaciones y son utilizadas en pacientes que requieren soporte transfusional prolongado. ${ }^{26}$

\section{Manejo del paciente con refractariedad plaquetaria}

Existen tres estrategias para brindar terapia transfusional a los pacientes con aloinmunización plaquetaria: el fenotipo HLA parecido o igual al receptor (HLA matching), la prueba cruzada negativa (cross matching plaquetario) y la predicción específica de anticuerpos (antibody specificity prediction). ${ }^{18}$

\section{Transfusión de plaquetas HLA compatibles}

El fenotipo HLA compatible es la técnica más recomendada, teniendo en cuenta que la mayoría de los casos es explicada por estos anticuerpos. Es efectiva en 70 a $80 \%$ de los casos, siendo las principales causas de su fracaso la presencia de anticuerpos antiplaquetarios, aloanticuerpos contra HLA C o del completo de histocompatibilidad menor ( $E, F, G)$, que no son tipificados rutinariamente, o la elección de transfusión de unidades parcialmente compatibles. ${ }^{7,15,18}$

La selección de plaquetas HLA compatibles se basa en el conteo del número de antígenos en los que difiere el donante del receptor, tipo HLA A y B; la mayor limitación radica en la necesidad de tipificar al paciente antes de iniciar una búsqueda dirigida de donante compatible, lo que requiere un gran grupo de donantes para identificar una unidad compatible (al menos 3,000 donantes tipificados para encontrar un grado de compatibilidad BX o mayor). ${ }^{15}$

Los antígenos del HLA-1 se denominan antígenos privados, ya que sólo están presentes en un individuo, pero existe desarrollo de anticuerpos contra moléculas que no están en el HLA-1, con similitud estructural a los antígenos del HLA que se denominan antígenos públicos (CRECS, por sus siglas en inglés). La incompatibilidad generada por estos aloanticuerpos también debe tenerse en cuenta al elegir las unidades plaquetarias para aplicar. ${ }^{16,18}$

De forma usual, se aceptan trasfusiones hasta un grado de compatibilidad BX, siendo el mejor grado el A. Además, una de las ventajas que provee la transfusión de plaquetas HLA compatibles es disminuir la posibilidad de sensibilización en el receptor hacia nuevos antígenos. ${ }^{18}$

\section{Transfusión de plaquetas por prueba cruzada}

Esta estrategia de selección de plaquetas tiene como principio el escoger una unidad compatible con el receptor a través de la incubación de las plaquetas del posible donante con el plasma del receptor. Es una prueba rápida en comparación con el tiempo que implica la fenotipificación del HLA; sin embargo, debe ser realizada periódicamente, debido a la vida útil de la plaqueta que es de cinco días aproximadamente. De manera adicional, la selección de plaquetas por prueba cruzada no elimina la posibilidad de sensibilización a nuevos antígenos por parte del receptor. ${ }^{7,16,18}$

La transfusión de plaquetas por prueba cruzada ha mostrado utilidad a la hora de incrementar el conteo plaquetario; no obstante, no hay evidencia de su impacto en disminuir hemorragias ni la mortalidad. ${ }^{26}$ 
De forma usual las plataformas tienen la posibilidad de evaluar hasta 94 unidades y se pueden valorar diferentes pacientes en la misma plataforma, las técnicas tienden a ser sencillas y ágiles, lo que permite su empleo en escenarios de transfusión prioritaria. ${ }^{27}$

Considerando que la compatibilidad con el HLA C y el complejo menor de histocompati- bilidad no se hace rutinariamente, las pruebas cruzadas permiten valorar la reactividad de aloanticuerpos contra estos antígenos y así ajustar la terapia transfusional. ${ }^{28}$

La posibilidad de usar sangre total y sistemas automatizados disponibles en la actualidad, para lograr mínima manipulación y contaminación, permite maximizar la utilidad de estas pruebas. ${ }^{27}$

Tabla 2: Estrategias y alternativas de manejo.

\begin{tabular}{|c|c|c|c|}
\hline Tratamiento & Referencia & Hallazgos & Conclusión \\
\hline Antifibrinolíticos & $7,29,30$ & $\begin{array}{l}\text { Los estudios que han usado ácido aminoca- } \\
\text { proico o ácido tranexámico sugieren disminu- } \\
\text { ción del sangrado y necesidad de transfusión } \\
\text { de plaquetas }\end{array}$ & $\begin{array}{l}\text { Pueden ser usados como tera- } \\
\text { pia adyuvante en sangrados de } \\
\text { la mucosa }\end{array}$ \\
\hline $\begin{array}{l}\text { Inmunoglobulina } \\
\text { endovenosa }\end{array}$ & $7,31,32$ & $\begin{array}{l}\text { La evidencia no soporta su uso rutinario. Pue- } \\
\text { de tener alguna importancia en pacientes con } \\
\text { trombocitopenia severa inmune, en quienes } \\
\text { hayan fallado otras alternativas }\end{array}$ & $\begin{array}{l}\text { No de forma rutinaria, no } \\
\text { reemplaza el uso de plaquetas } \\
\text { HLA compatibles }\end{array}$ \\
\hline Esplenectomía & 15,33 & No tiene impacto en el CCl postransfusión & No recomendada \\
\hline Anti-Rh (D) & 15,34 & $\begin{array}{l}\text { No ha mostrado que disminuya la tasa de } \\
\text { refractariedad ni el requerimiento transfusional } \\
\text { plaquetario. } \\
\text { Incrementa el requerimiento transfusional de } \\
\text { glóbulos rojos }\end{array}$ & No recomendada \\
\hline $\begin{array}{l}\text { Adsorción en colum- } \\
\text { nas de proteína A }\end{array}$ & 35,36 & $\begin{array}{l}\text { Esta terapia fue efectiva en mejorar la respues- } \\
\text { ta plaquetaria en } 6 \text { de } 10 \text { pacientes de un estu- } \\
\text { dio; sin embargo, no tuvieron seguimiento }\end{array}$ & $\begin{array}{l}\text { Evidencia no clara. Esta tec- } \\
\text { nología no está disponible en } \\
\text { muchos centros. } \\
\text { Tiene potenciales efectos } \\
\text { adversos }\end{array}$ \\
\hline $\begin{array}{l}\text { Transfusión de } \\
\text { grandes dosis de } \\
\text { plaquetas }\end{array}$ & 35,37 & $\begin{array}{l}\text { La realización de transfusiones plaquetarias a } \\
\text { grandes dosis, parece que logra absorber una } \\
\text { gran cantidad de aloanticuerpos, llevando } \\
\text { a mejorar en las siguientes transfusiones los } \\
\text { síntomas de sangrado }\end{array}$ & $\begin{array}{l}\text { Puede considerarse en caso de } \\
\text { emergencia cuando no hay dis- } \\
\text { ponibles unidades de plaquetas } \\
\text { compatibles }\end{array}$ \\
\hline $\begin{array}{l}\text { Transfusión plaqueta- } \\
\text { ria sólo terapéutica }\end{array}$ & $35,38,39$ & $\begin{array}{l}\text { Restringir o suprimir las transfusiones profilác- } \\
\text { ticas y en ese caso, transfundir plaquetas pero } \\
\text { con un sentido terapéutico, empleando dosis } \\
\text { mayores a las habituales o con una frecuencia } \\
\text { mayor }\end{array}$ & No recomendado de rutina \\
\hline $\begin{array}{l}\text { Transfusión plaqueta- } \\
\text { ria lenta }\end{array}$ & 40 & $\begin{array}{l}\text { Anécdota exitosa en } 3 \text { pacientes, transfusión } \\
\text { en } 6 \text { horas }\end{array}$ & $\begin{array}{l}\text { Evidencia anecdótica, puede } \\
\text { ser útil en mantener la inte- } \\
\text { gridad vascular, sin evidencia } \\
\text { de incremento en el conteo } \\
\text { plaquetario }\end{array}$ \\
\hline Factor VII activado & $7,41,42$ & Control de sangrado & $\begin{array}{l}\text { Evidencia anecdótica, puede } \\
\text { considerarse en emergencias } \\
\text { por hemorragia }\end{array}$ \\
\hline
\end{tabular}




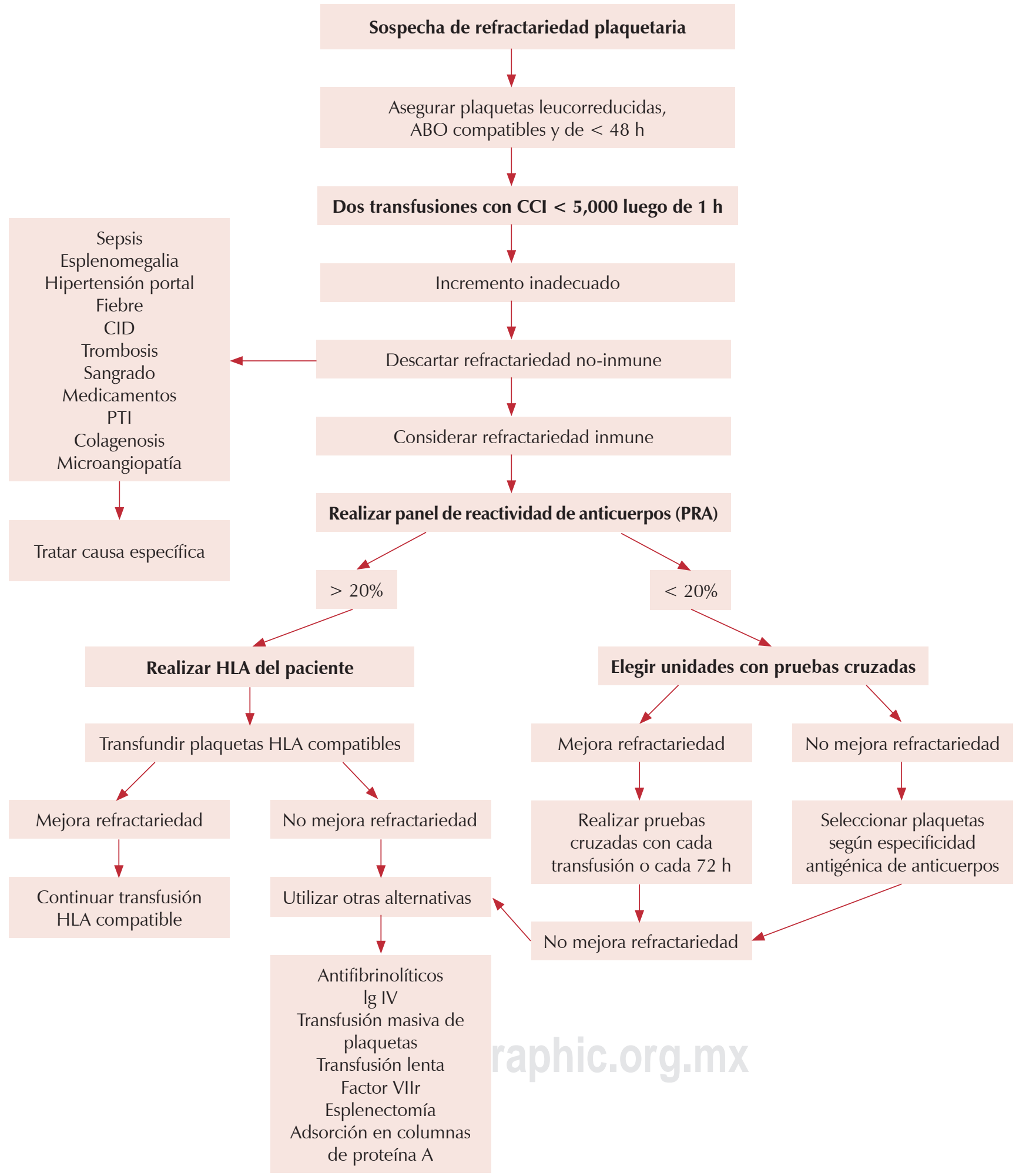

Figura 1: Algoritmo de manejo de refractariedad plaquetaria. 


\section{Transfusión de plaquetas seleccionadas por predicción específica de anticuerpos}

Es una tercera estrategia para el manejo de aloinmunización, es similar al enfoque rutinario usado para la aloinmunización de glóbulos rojos. Se hace una identificación de los anticuerpos del receptor y se proveen plaquetas de donantes que carezcan del antígeno contra el que está dirigido el anticuerpo del receptor. . $1,16^{-16}$

Este método no requiere una completa compatibilidad, sólo se rastrean los antígenos contra los que están dirigidos los aloanticuerpos del receptor, lo que aumenta el número de donantes de forma notable. ${ }^{15,18}$ Sin embargo, es un estudio que debe hacerse periódicamente al receptor por la posibilidad de aloinmunización a nuevos antígenos del donante, con el tiempo y con el uso prolongado de transfusiones. Demanda tiempo, en comparación con las pruebas cruzadas, por lo que debe conocerse con antelación los aloanticuerpos del receptor para iniciar la búsqueda de un donante. Adicionalmente no es útil para tipificación de antígenos plaquetarios. ${ }^{15,18}$

\section{Otras estrategias}

Se encuentran disponibles otras estrategias para el manejo de los pacientes con refractariedad plaquetaria; sin embargo, ninguna de estas ha probado ser exitosa (Tabla 2).

\section{Conclusiones}

El manejo de la refractariedad plaquetaria inicia con la prevención de la sensibilización. A la fecha no existe un método para diagnosticar de forma precisa la refractariedad de origen no inmune, y en muchas ocasiones puede coexistir con algún grado de refractariedad inmune. En cuanto al manejo de la refractariedad no inmune, debe establecerse la causa de base y tratarla, el uso de terapia inmunosupresora puede ser útil en presencia de autoanticuerpos, en contraste con la refractariedad inmune, en la cual el uso de la terapia inmunosupresora no ha demostrado un rol relevante.

Por lo tanto, se propone el siguiente algoritmo de manejo para la refractariedad plaquetaria (Figura 7).

\section{Referencias}

1. Fasano R, Josephson C. Platelet transfusion goals in oncology patients. Hematology. 2015; 2015: 462-470. doi: 10.1182/ asheducation-2015.1.462.

2. Gibson BE, Todd A, Roberts I et al. Transfusion guidelines for neonates and older children. Br J Haematol. 2004; 124 (4): 433-453. doi: 10.1111/j.1365-2141.2004.04815.x.

3. Wandt H, Ehninger G, Gallmeier W. New strategies for prophylactic platelet transfusion in patients with hematologic diseases. Oncologist. 2001; 6 (5): 446-450. doi: 10.1634/theoncologist.6-5-446.

4. Phekoo K, Hambley H, Schey S, Win N, Carr R, Murphy M. Audit of practice in platelet refractoriness. Vox Sanguinis. 1997; 73 (2) 81-86. doi: 10.1046/j.1423-0410.1997.7320081.x.

5. The Trial to Reduce Alloimmunization To Platelets Study Group. Leukocyte reduction and utraviolet $B$ irradiation of platelets to prevent alloimmunization and refractoriness to platelet transfusions. N Engl J Med. 1997; 337 (26): 1861-1869.

6. Slicther S, Davis K, Enright H, Braine H, Gernshimer T, Kao K et al. Factors affecting post-transfusion platelets increments, platelet refractoriness, and platelet transfusion intervals in thrombocytopenic patients. Blood. 2005; 105 (10): 4106-4114. doi: 10.1182/blood-2003-08-2724.

7. Quillen K. Diagnosis and management of platelet alloimmunization. Column Ask the Hematologist. Hematology. 2013; 10 (6): 1023.

8. Daly A, Hasegawa W, Lipton J, Messner H, Kiss T. Transplantationassociated thrombotic microangiopathy is associated with transplantation from unrelated donors, acute graft-versus-host disease and veno-occlusive disease of the liver. Transfus Apher Sci. $2002 ; 27$ (1): 3-12.

9. McCullough J. Overview of platelet transfusion. Semin Hematol. 2010; 47 (3): 235-242. doi: 10.1053/j.seminhematol.2010.04.001.

10. Novotny V. Prevention and management of platelet transfusion refractoriness. Vox Sang. 1999; 76 (1): 1-13.

11. Schiffer C, Anderson K, Bennett C, Bernstein S, Elting L, Goldsmith $M$ et al. Platelet transfusion for patients with cancer: clinical practice guidelines of the American Society of Clinical Oncology. J Clin Oncol. 2001; 19 (5): 1519-1538.

12. Pavensky K, Freedman J, Semple J. HLA alloimmunization against platelet transfusions: pathophysiology, significance, prevention and management. Tissue Antigens. 2012; 79 (4): 237-245. doi: 10.1111/.1399-0039.2012.01852.x.

13. Dzik S. How I do it: platelet support for refractory patients. Transfusion. 2007; 47 (3): 374-378. doi: 10.1171/.j.1537-2995.2007.01126.x.

14. Delaflor-Weiss E, Mintz PD. The evaluation and management of platelet refractoriness and alloimmunization. Transfus Med Rev. 2000; 14 (2): 180-196. doi: 10.1016/s0887-7963(00)80007-3.

15. Hod E, Schwartz J. Platelet transfusion refractoriness. $\mathrm{Br} \mathrm{J}$ Haematol. 2008; 142 (3):348-360. doi:10.1111/.1365-2141.2008.07189.x.

16. Stanworth S, Navarrete C, Estcourt L, Marsh J. Platelet refractoriness-practical approaches and ongoing dilemmas in 
patient management. Br J Haematol. 2015; 171 (3): 297-305. doi: 10.1111/bjh.13597.

17. Schiffer C. State-of-the-art mini-review. Management of patients refractory to platelet transfusion. Leukemia. 2001; 15 (4): 683-685.

18. Forest $\mathrm{S}, \mathrm{Hod} \mathrm{E}$. Management of the platelet refractory patient. Hematol Oncol Clin N Am. 2016; 30 (3): 665-677. doi: 10.1016/j. hoc.2016.01.008.

19. Valsami S, Dimitroulis D, Gialeraki A, Chimonidou M, Politou $M$. Current trends in platelet transfusions practice: the role of $\mathrm{ABO}-\mathrm{RhD}$ and human leukocyte antigen incompatibility. Asian J Transfus Sci. 2015; 9 (2): 117-123. doi: 10.4103/09736247.162684.

20. Kiefel V, König C, Kroll H, Santoso S. Platelet alloantibodies in transfused patients. Platelet alloantibodies in transfused patients. Transfusion. 2001; 41 (6): 766-770. doi: 10.1046/j.15372995.2001.41060766.x.

21. Panzer S, Auerbach L, Cechova E, Fischer G, Holesteiner A, Kitll E et al. Maternal alloimmunization against fetal platelet antigens: a prospective study. Br J Haematol. 1995; 90 (3): 655-660.

22. Kurz M, Greinix H, Cker P, Kalhs P, Kno P, Mayr W et al. Specificities of anti-platelet antibodies in multitransfused patients with haemato-oncological disorders. Br J Haematol. 1996; 95 (3): 564 569. doi: 10.1046/j.1365-2141.1996.d01-1936.x.

23. Dutcher J, Schiffer C, Aisner J, Wiernik P. Long-term followup of patients with leukemia receiving platelet transfusions: identification of a large group of patients who do not become alloimmunized. Blood. 1981; 59 (5): 1007-1011.

24. Seftel M, Growe G, Petraszko T, Barret B, Le A, Lee C et al. Universal leukorecution in Canada decreases platelet alloimmunization and refractoriness. Blood. 2004; 103 (1): 333-339. doi: 10.1182/ blood-2003-03-0940.

25. Heddle N, Arnold D, Boye D, Webert K, Resz I, Dumont L. Comparing the efficacy and safety of apheresis and whole bloodderived platelet transfusions: a systematic review. Transfusion. 2008; 48 (7): 1447-1458. doi: 10.1111/j.1537-2995.2008.01731.x.

26. Vasallo R, Fung M, Rebulla P, Duquesnoy R, Saw C, Slichter S. Utility of cross-matched platelet transfusions in patients with hypoproliferative thrombocytopenia: a systematic review. Transfusion. 2014; 54 (4): 1180-1191. doi: 10.1111/trf.12395.

27. Rebulla P, Morelati F, Revelli N, Villa M, Paccapelo C, Nocco A. Outcomes of an automated procedure for the selection of effective platelets for patients refractory to random donors based on crossmatching locally available platelet products. $\mathrm{Br} J$ Haematol. 2004; 125 (1): 83-89.

28. Kopko P, Warner P, Kresie L, Pancoska C. Methods for the selection of platelet products for alloimmune-refractory patients. Transfusion. 2015; 55 (2): 235-244. doi: 10.1111/trf.12921.

29. Kalmadi S, Tiu R, Lowe C. Epsilon aminocaproic acid reduces transfusion requirements in patients with thrombocytopenic hemorrhage. Cancer. 2006; 107 (1): 136-140.
30. Antun A, Gleason S, Arellano M. Epsilon aminocaproic acid prevents bleeding in severely thrombocytopenic patients with hematological malignancies. Cancer. 2013; 119 (21): 3784-3787.

31. Ratko T, Burnett D, Foulke $\mathrm{C}$. Recommendations for off-label use of intravenously administered immunoglobulin preparations. University Hospital Consortium Expert Panel for Off-Label Use of Polyvalent Intravenously Administered Immunoglobulin Preparations. JAMA. 1995; 273 (23): 1865-1870.

32. Schiffer C, Hogge D, Aisner J. High-dose intravenous gammaglobulin in alloimmunized platelet transfusion recipients. Blood. 1984; 64 (4): 937-940.

33. Hogge D, Dutcher J, Aisner J. The ineffectiveness of random donor platelet transfusion in splenectomized, alloimmunized recipients. Blood. 1984; 64 (1): 253-256.

34. Heddle N, Klama L, Kelton J. The use of anti-D to improve posttransfusion platelet response: a randomized trial. $\mathrm{Br} \mathrm{J}$ Haematol. 1995; 89 (1): 163-168.

35. Muñiz E, Martínez C, Madoz P. Refractariedad a las transfusiones de plaquetas. Med Clin. 2003; 120 (14): 544-549.

36. Christie D, Howe R, Lennon S, Sauro S. Treatment of refractoriness to platelet transfusion by protein A column therapy. Trans. 1993; 33 (3): 234-242.

37. Nagasawa T, Kim B, Baldini M. Temporary suppression of circulating antiplatelet alloantibodies by the massive infusion of fresh, stored, or lyophilized platelets. Trans. 1978; 18 (4): 429-435.

38. Friedmann A, Sengul H, Lehmann H, Schwartz C, Goodman S. Do basic laboratory tests or clinical observations predict bleeding in thrombocytopenic oncology patients? A reevaluation of prophylactic platelet transfusions. Transfus Med Rev. 2002; 16 (1): 34-45.

39. Lee E, Schiffer C. Serial measurement of Iymphocytotoxic antibody and response to nonmatched platelet transfusion in alloimmunized patients. Blood. 1987; 70 (6): 1727-1729.

40. Narvios A, Reddy V, Martinez F. Slow infusion of platelets: a possible alternative in the management of refractory thrombocytopenic patients. Am J Hematol. 2005; 79 (1): 80.

41. Heuer L, Blumenberg D. Management of bleeding in a multitransfused patient with positive HLA class I alloantibodies and thrombocytopenia associated with platelet dysfunction refractory to transfusion of cross-matched platelets. Blood Coagul Fibrinol. 2005; 16 (4): 287-290.

42. Vidarsson B, Onundarson P. Recombinant factor VIla for bleeding in refractory thrombocytopenia. Thromb Haemost. 2000; 83 (4): 634-635. 